\title{
Preparation and Reactivity of Nanocrystalline Cocrystals Formed via Sonocrystallization
}

\author{
Dejan-Krešimir Bučar and Leonard R. MacGillivray* \\ Department of Chemistry, University of Iowa, Iowa City, IA 52242-1294 \\ (USA), Fax: (+1) 319-335-1270
}

\section{Supporting Information}

Figure S1. X-ray powder patterns of 2(resorcinol) 2(4,4'-bpe).

Figure S2. ${ }^{1}$ H-NMR spectrum of 2(resorcinol) $2(4,4$ '-bpe) (macrococrystals before UVirradiation).

Figure S3. ${ }^{1} \mathrm{H}-\mathrm{NMR}$ spectrum of 2(resorcinol) $2(4,4$ '-bpe) (macrococrystals after UVrradiation).

Figure S4. ${ }^{1} \mathrm{H}-\mathrm{NMR}$ spectrum of 2(resorcinol)-2(4,4'-bpe) (nanostructured cocrystals before UV-irradiation).

Figure S5. ${ }^{1} \mathrm{H}-\mathrm{NMR}$ spectrum of 2(resorcinol) $2\left(4,4^{\prime}\right.$-bpe) (nanostructured cocrystals after UV-irradiation). 
Figure S1. X-ray powder patterns of 2(resorcinol)-2(4,4'-bpe): (1) calculated, (2) macrococrystals (before UV-irradiation), (3) nanostructured cocrystals (before UVirradiation) and 2(resorcinol) (4,4'-tpcb): (4) macrococrystals (after UV-irradiation), (5) nanostructured cocrystals (after UV-irradiation).

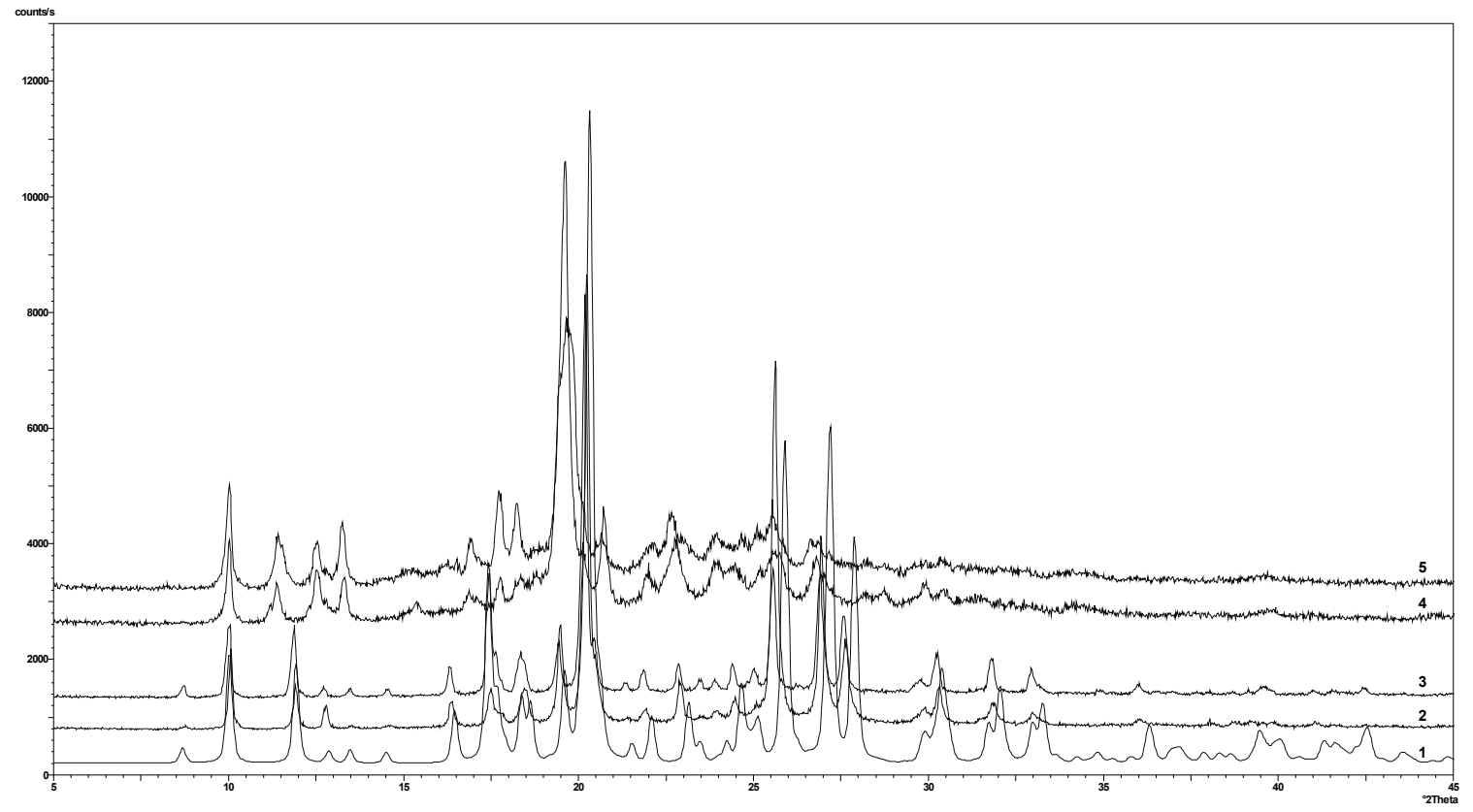


Figure S2. ${ }^{1} \mathrm{H}-\mathrm{NMR}$ spectrum of 2(resorcinol) $2\left(4,4^{\prime}\right.$-bpe) (macrococrystals before UVirradiation).

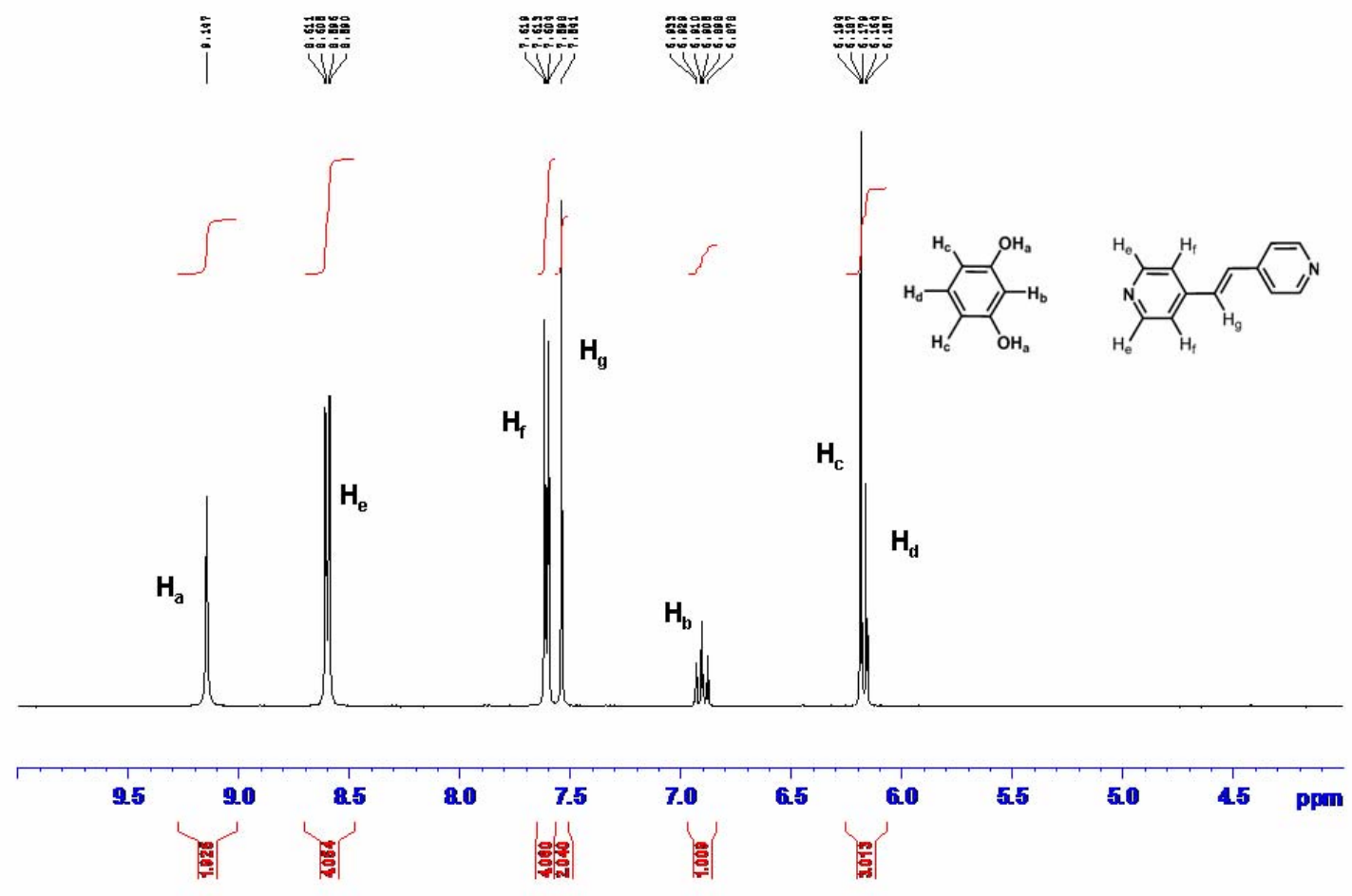


Figure S3. ${ }^{1} \mathrm{H}-\mathrm{NMR}$ spectrum of 2(resorcinol) $2\left(4,4^{\prime}\right.$-bpe) (macrococrystals after UVirradiation).

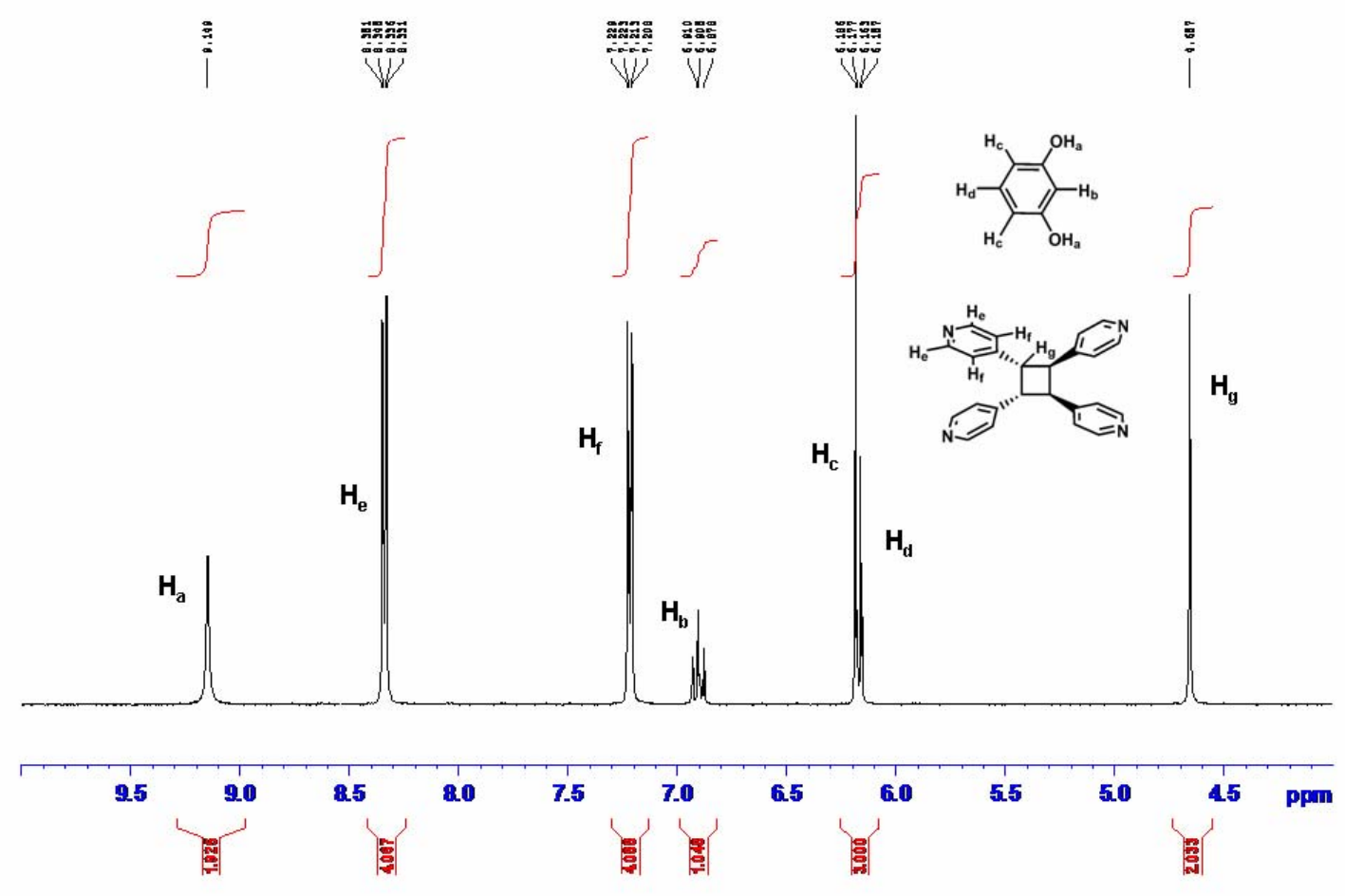


Figure S4. ${ }^{1}$ H-NMR spectrum of 2(resorcinol) $2\left(4,4^{\prime}\right.$-bpe) (nanostructured cocrystals before UV-irradiation).

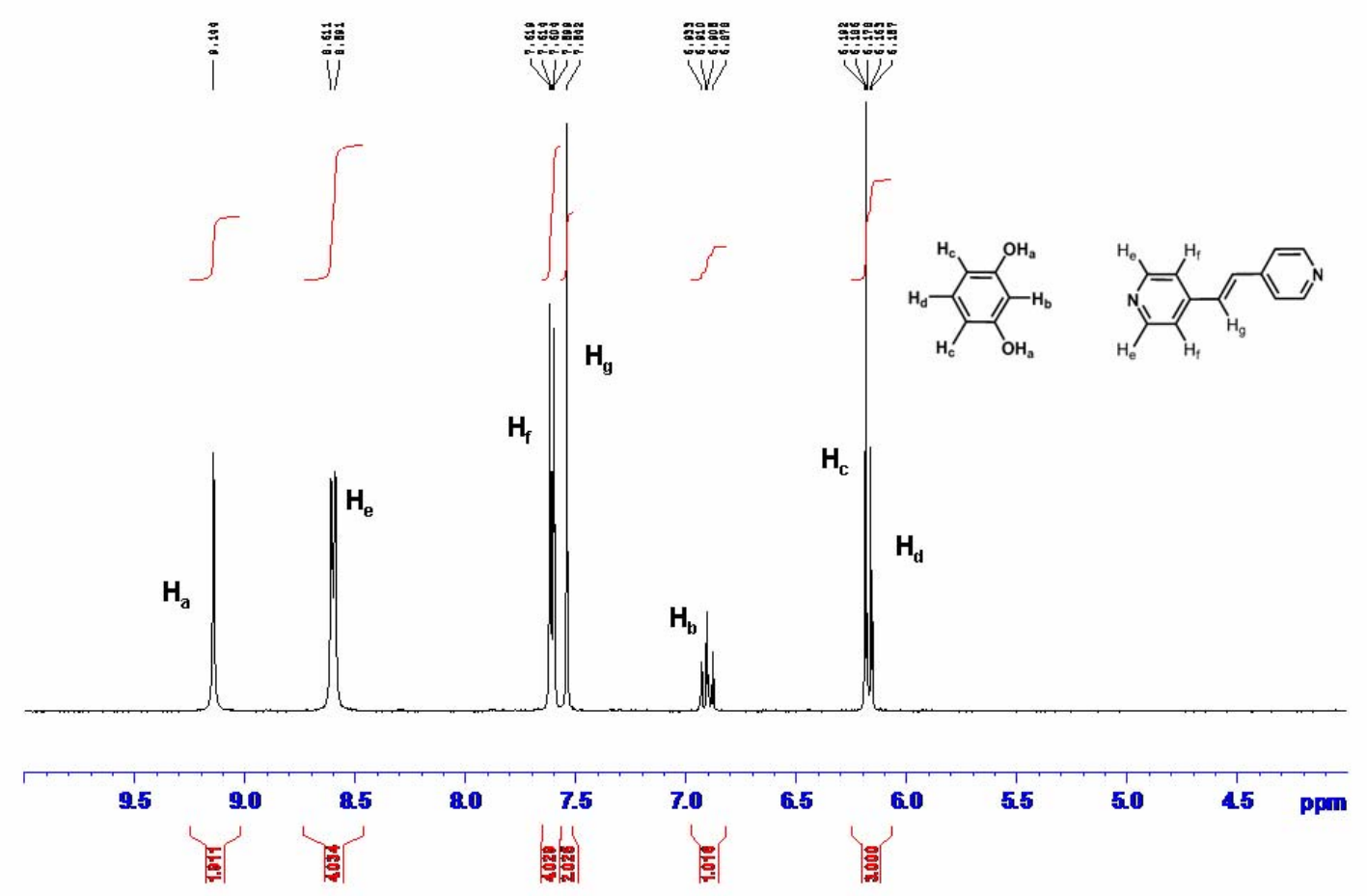


Figure S5. ${ }^{1} \mathrm{H}-\mathrm{NMR}$ spectrum of 2(resorcinol) $2\left(4,4^{\prime}\right.$-bpe) (nanostructured cocrystals after UV-irradiation).

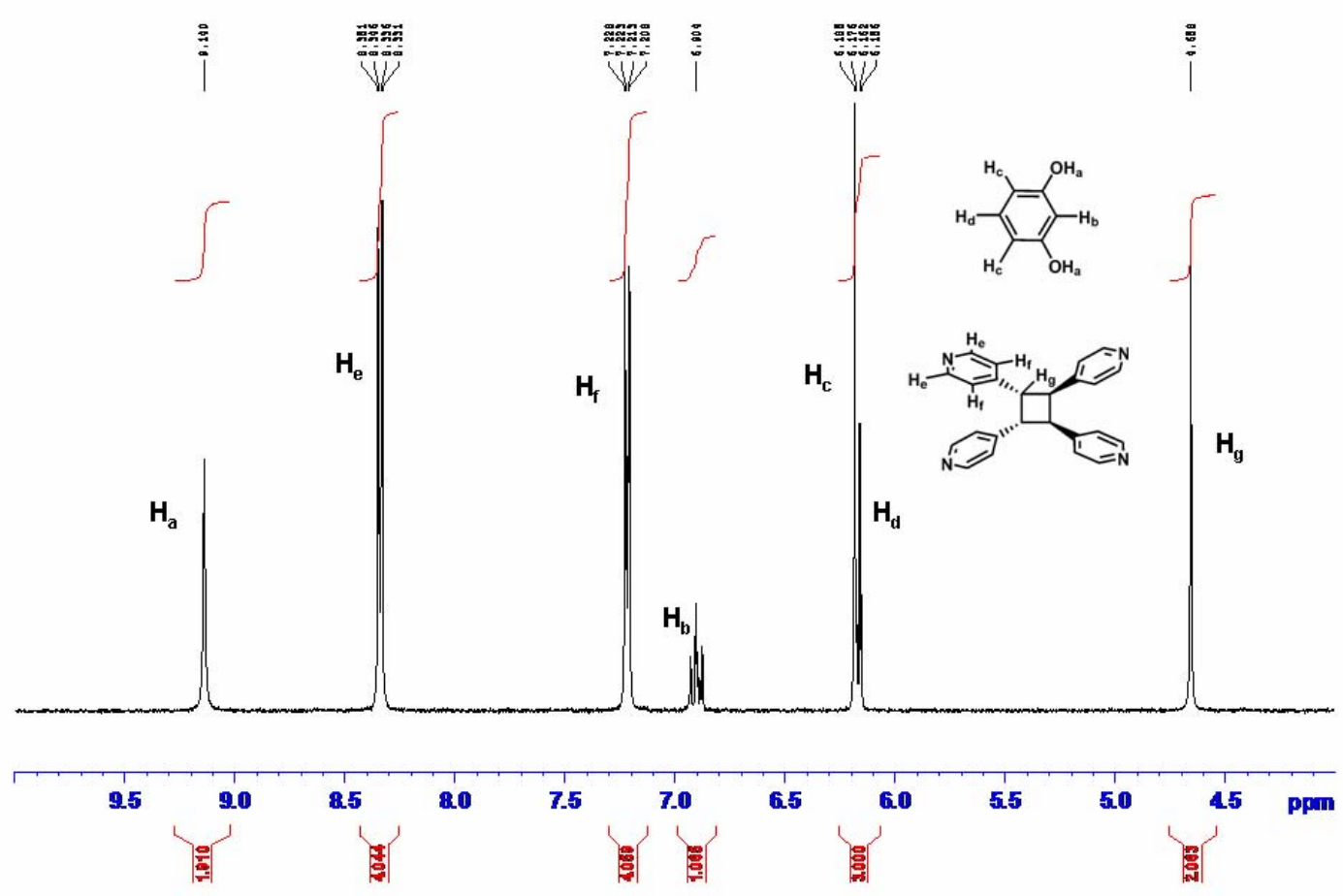

